\title{
Applied Research In Technique Of Intensity Modulated Radiation Therapy (Jo-Imrt) In Dongnai Generai Hospital
}

\author{
Dương Thanh Tài ${ }^{1}$, Đinh Thanh Bình ${ }^{1}$,Trương Thiết Dũng ${ }^{1}$, \\ Nguyễn Văn Minh ${ }^{1}$, Trần Phạm Ngọc Trinh ${ }^{1}$ \\ Le Ba Loc ${ }^{2}$, Nguyen Van Hai ${ }^{2}$, Nguyễn Văn Hùng ${ }^{3}$ \\ Dongnai General Hospital ${ }^{1}$ Dalat Vocational Training College ${ }^{2}$ Vietnam Atomic Energy Institute ${ }^{3}$
}

\begin{abstract}
Intensity modulated radiation therapy (IMRT) is a modern technique in cancer treatment that radiation dose is more closely shaped to fit the tumour and spared nearby critical normal tissues. This technique has been recognized and widely used in clinical applications by linear accelerators (LINAC) with multi-leaf collimator (MLC) systems in developed countries. However, almost LINAC without MLC have still been used most countries, but it could be applied this technique for LINACs with jaws that are not to need use of MLC. The aim of this study is to apply IMRT technique by Prowess Panther code for cancer patients in Dongnai General Hospital. This is particularly significant in both society and economy in difficult conditions of our country due to high cost of LINACs with MLCs. Besides, the climate has been hot and wet in Vietnam, so operation and maintenance for the LINAC with MLCs has been very difficult.
\end{abstract}

Keywords: - Intensity modulated radiation therapy (IMRT), Cancer, Linear electron accelerator (LINAC), Multi-leaf collimator (MLC).

\section{INTRODUCTION}

Intensity modulated radiation therapy (IMRT) is becoming the standard care for many tumor sites/types. IMRT is delivered with mostly multi-leaf collimator (MLC), and occasionally with compensators. Recently, investigators are also exploring the use of independent Jaws, an alternative to MLC and compensators, to deliver IMRT $[4,8]$. It has been shown that the Jaw-only IMRT (JO-IMRT) is capable of generating conformal dose distributions comparable to those generated by MLC. Very recently, JO-IMRT is implemented in a commercial system (Panther, Prowess Inc.) using direct aperture optimization (DAO) algorithm, the optimizer in our approach directly produces apertures using conventional jaws with optimized weighting factors $[7,8]$.

Although Intensity-modulated radiotherapy (IMRT) has been widely implemented for many medical centers, many radiation therapy centers worldwide still use cobalt -60 machines or early versions of linear accelerators without multi-leaf collimator (MLC). For these centers, a simple collimator with conventional jaws (or independent jaws) would be much more affordable and easier to maintain. The aim of this study was to investigate the implementation of JO-IMRT plans and quality assurance (QA) of JO-IMRT plans in order to realize increased efficiency of utilization of therapy facilities.

A major limitation of the efficacy of radiotherapy treatment is the production of undesirable complication by the irradiation of healthy tissue inherent in a given radiotherapy technique. Many organs are relatively sensitive to radiation damage ( the spinal cord, parotid gland and the eyes are common examples) and must be given special consideration during radiotherapy treatment planning. In general, treatment planners attempt to optimize the dose distribution achievable with a given treatment strategy to deliver a tumorcidal dose of radiation to a target volume while minimizing the amount of radiation absorbed in healthy tissue outside the target volume. Conventional treatment strategies use limited number of shaped beams and restrict the orientation of the beams to coplanar fields. Using JO-IMRT method helps reject these weak points. In this paper, we conducted a planning study for nasopharynx patient case. For this patient, two plans were generated using conventional jaws (jaw only) and the conventional 3D conformal planning technique and each JO-IMRT plan will be QA to prove accuracy and reliability of this method

\section{MATERIAL AND METHODS}

A commercial treatment planning system (Panther Ver 4.6, Prowess Inc.) including DAO algorithm for IMRT inverse planning is used to generate JO-IMRT plans. IMRT QA measurement was carried out using a cylindrical phantom built with water-equivalent material. Three chambers can be inserted into the phantom at different locations. In this work, a farmer type chamber was placed at isocenter or several off-center locations to measure the absolute doses. 


\section{Case Nasopharynx case ( DTM)}

\section{RESULTS}

Critical structures: Parotid gland and brain stem. The volume to be irradiated isn't include cervical lymphatic. For this case, 7 beams/49 segments were used. The dose computation took approximately $1.5 \mathrm{~h}$. The gross tumor volume (GTV) which prescribed target dose for this case was $200 \mathrm{cGy}$ for 33 fractions.

Figure 1,2 provide dose volume histogram (DVH) and isodose distribution comparisons between the JO-IMRT and 3D-CRT
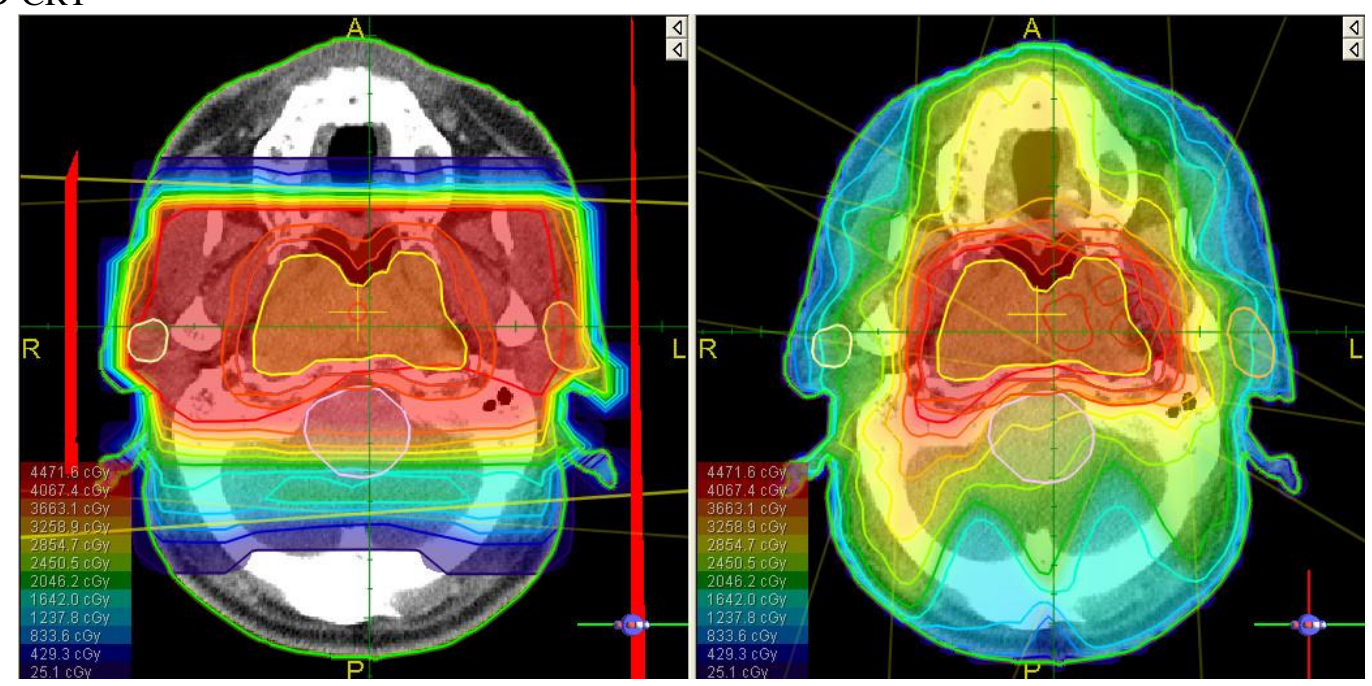

Fig. 1: Comparison of isodose distributions between 3D-CRT and JO-IMRT plans for nasopharynx patient.

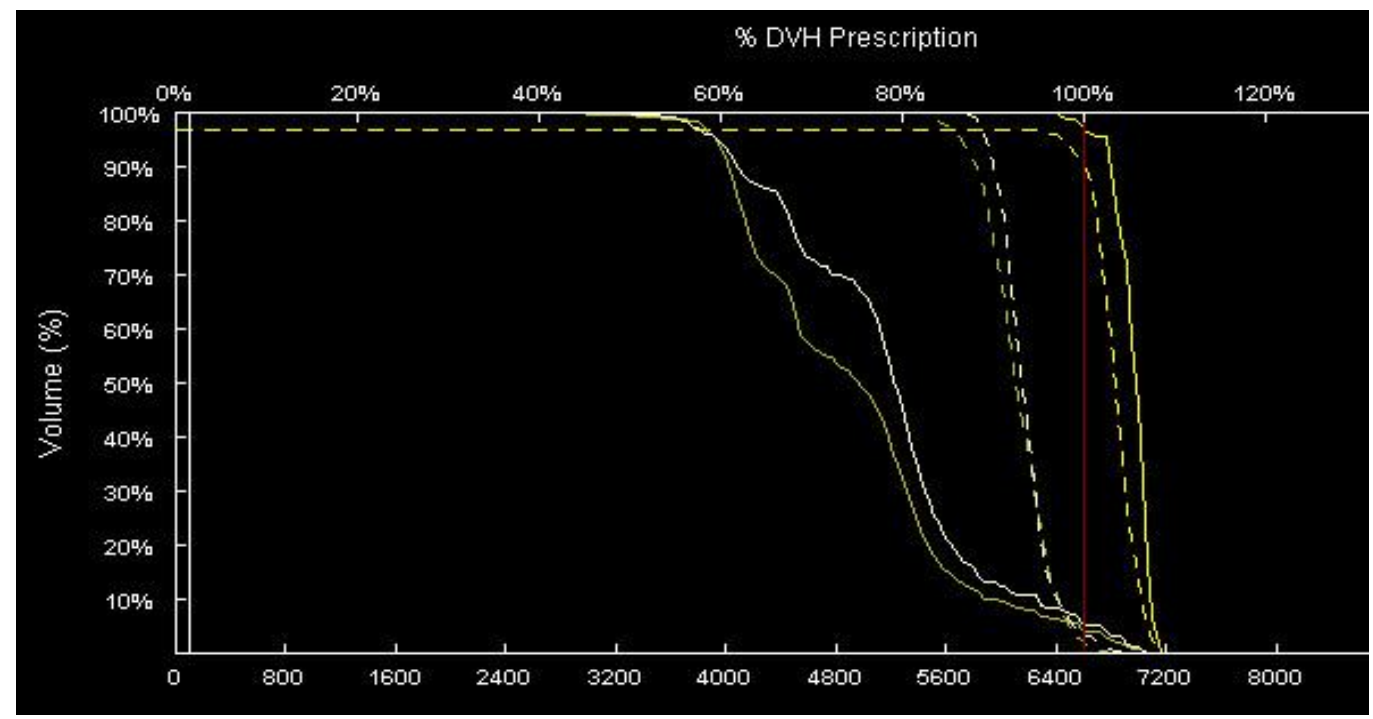

Fig. 2. Dose volume histogram for the nasopharynx patient. Dashed lines denote the JO-IMRT plan, whereas the Solid lines denote 3D-CRT plan

It can be seen that the Jaw-Only approach provided much better plan quality than achieved by 3D-CRT. The dose to Spinal cord and parotid gland in JO-IMRT plan is less than in 3D-CRT plan. This plan was delivered in approximately 8 minutes on a Siemens Primus Linac

\begin{tabular}{|l|c|c|}
\hline \multirow{2}{*}{\multicolumn{1}{|c|}{ Treated volume }} & \multicolumn{2}{c|}{ Total Dose (cGy) } \\
\cline { 2 - 3 } & 3D-RT & IMRT \\
\hline PTV & 6935 & 6807 \\
\hline Spinal cord & 4485 & 4347 \\
\hline Parotid gland (Right) & 6531 & 3373 \\
\hline Parotid gland (Left) & 6131 & 3138 \\
\hline
\end{tabular}


After optimization, the treatment parameters for the JODAO case were transferred to the control computer of our Siemens linear accelerator. We then delivered the plan to the cylindrical phantom and measured absolute dose with an ionization chamber.

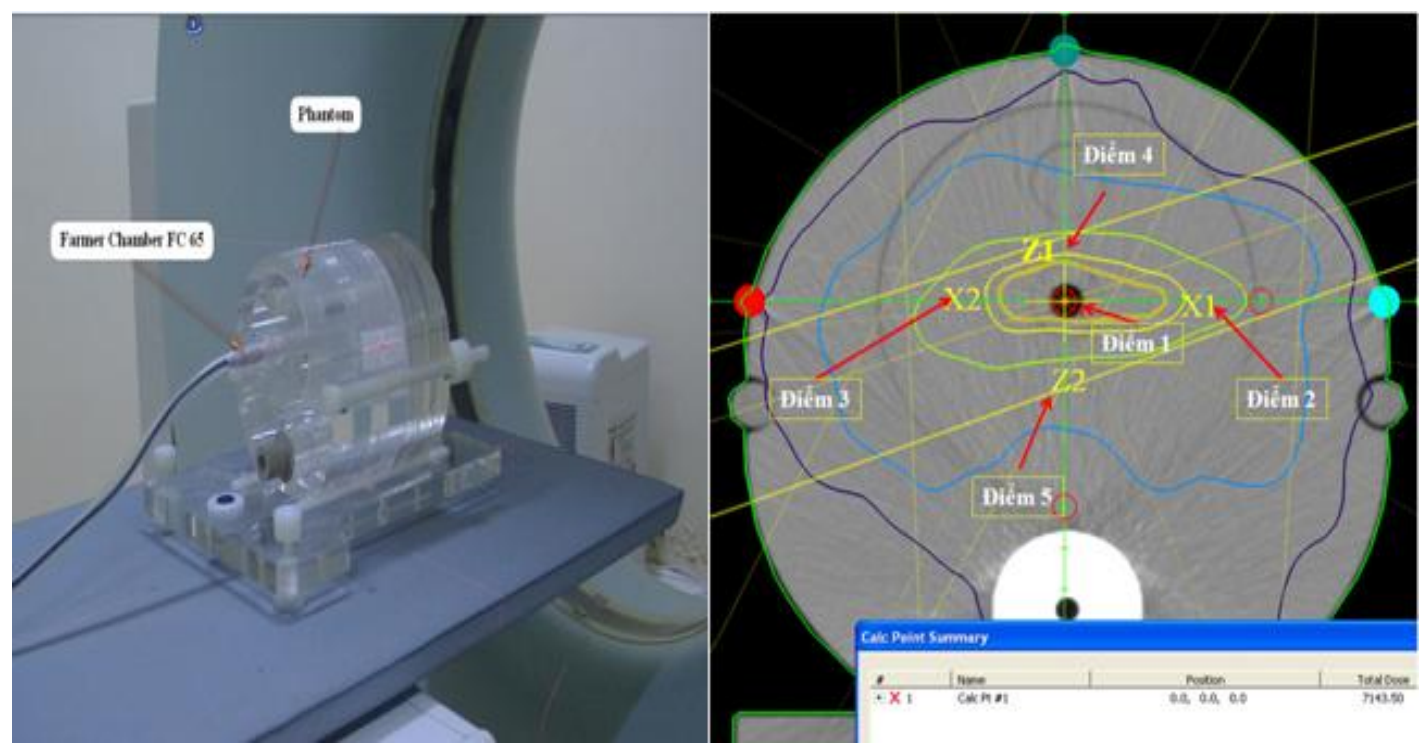

The dose distribution on cylindrical phantom at isocenter of case.

Table: The difference between the value of calculation and measurement

\begin{tabular}{|c|c|c|c|}
\hline Point & $\begin{array}{c}\text { Calculation } \\
(\mathbf{c G y})\end{array}$ & $\begin{array}{c}\text { Measurement } \\
(\mathbf{c G y})\end{array}$ & $\begin{array}{c}\text { Deviation } \\
(\mathbf{\%})\end{array}$ \\
\hline Point 1 & 1157,3 & 1158,0 & 0,1 \\
\hline Point 2 & 1159,5 & 1179,0 & 1,7 \\
\hline Point 3 & 1303,2 & 1327,0 & 1,8 \\
\hline Point 4 & 1728,4 & 1741,0 & 0,7 \\
\hline Point 5 & 1408,6 & 1397,0 & $-0,8$ \\
\hline Point 6 & 1076,0 & 1077,0 & 0,1 \\
\hline Point 7 & 1741,4 & 1698,0 & $-2,5$ \\
\hline
\end{tabular}

The absolute dose measurement was $2.5 \%$ different from that predicted by TPS- Prowess Panther. The plan was delivered in approximately 8 min on a Siemens Primus Linac

\section{DISCUSSION AND CONCLUSION}

The JO-IMRT plans generated with the DAO algorithm using a commercial treatment planning system are superior than the corresponding 3DCRT plans and are clinically acceptable in terms of their dosimetric feature (e.g., conformity and/or uniformity). The JO-IMRT plans can be reliably delivered on Siemens Linac equipped with independent Jaws. The JO-IMRT has been introduced in Dong Nai Hospital as a result of this work.

Based on the results of radiotherapy planning with JO-IMRT compared with 3D-CRT, confirmed that the JO-IMRT has created the optimal dose distribution in the target volume and dose reduction for healthy bodies. The error results can be higher than large hospitals but the errors are less than 5\%. Here are the initial results of the study to implement JO-IMRT techniques in Dong Nai province general hospital in the near future. it is especially significant both economically and effectively treated. So now, this technique has only been applied at Bach Mai hospital and hospital K. 


\section{REFERENCES}

[1] Arno J. Mundt, John C. Roeske, (2005), Intensity modulated radiation therapy-a clinical perspective, BC Decker Inc, The United States of America.

[2] Nguyễn Thái Bình (2008), Method of IMRT optimization of shallow tumor cases where the PTV extends into the build up region, University of Cambridge.

[3] C. Yu, Christopher J. Amies (2008), "Planing and delivery of intensity modulated radiation therapy", Medical physics, Vo. 35, No 12.

[4] Dai J R and Hu Y M (1999), "Intensity-modulation radiotherapy using independent collimators: an algorithm study", Med.Phys, Vo. 26.

[5] Earl M A, Shepard D M, Naqvi S, Li X A and Yu C X (2003), "Inverse planning for intensity-modulated arc therapy using direct aperture optimization”, Phys. Med. Biol, Vo.48, 1075-89.

[6] Kestin L L, Sharpe M B, Franzier R C (2000), "Intensity modulation to improve dose uniformity with tangential breast radiotherapy: Initial clinical experience”, Radiat. Oncol. Biol. Phys, Vo. 48, 1559-68.

[7] M.A.Earl, D.M.Shepard (2002), "Direct aperture optimization: A turnkey solution for step-and-shoot IMRT", Medical physics, Vo. 29, No 6, 21201-1595.

[8] M. A. Earl, D. M. Shepard (2007), "Jaws only IMRT using direct aperture optimization", Medical physics, Vo. 34, No 1, 21201-1595.

[9] www.radiologyinfo.org, Intensity Modulated Radiation Therapy.

[10] www.mayoclinic.org, Intensity Modulated Radiation Therapy.

[11] www.medicalphysics.org, IMRT For Head And Neck Cancer. 